\title{
Kangaroo Mother Care versus Incubator in Transporting Stable Preterm Neonates: A Randomized Controlled Trial
}

\author{
Renelyn P. Ignacio, $\mathrm{MD}^{1}$ and Maria Esterlita T. Villanueva-Uy, MD, MSPH${ }^{2}$ \\ ${ }^{1}$ Newborn Intensive Care Unit, Department of Pediatrics, Baguio General Hospital and Medical Center \\ ${ }^{2}$ Department of Pediatrics, College of Medicine and Philippine General Hospital, University of the Philippines Manila
}

\begin{abstract}
Background. Transporting preterm neonates soon after birth entails risks. Only one study among many about the beneficial effects of Kangaroo Mother Care (KMC) had cited it as a safe and effective alternative to transport incubators.

Objective. To determine if $\mathrm{KMC}$ transport could be an alternative to transport incubators by comparing the physiological outcomes of the two transport methods.

Methods. This is a parallel non-blinded randomized-controlled trial funded by KMC Foundation, Philippines, Inc, of physiologically stable preterm neonates weighing $\leq 2200$ grams delivered at a tertiary government hospital from September 10, 2011, to April 18, 2012. After obtaining written consent from their mothers, participants were randomly assigned to either the intervention or control group. The intervention groups were transported from the delivery room to the NICU while on skin-to-skin contact with the caregiver. In contrast, those in the control group were placed in a transport incubator. Vital signs, oxygen saturation, and blood glucose were measured before transport and upon NICU arrival. Adverse effects were monitored. Data were recorded using a standard database.
\end{abstract}

Results. Ninety-two participants were recruited, forty-six in each arm. Two dropped out. The mean change in heart rate in the $\mathrm{KMC}$ transport decreased by 1.6 beats per minute; respiratory rate decreased by 0.18 breaths per minute, the temperature increased by $0.01^{\circ} \mathrm{C}$, oxygen saturation decreased by $0.07 \%$, blood glucose decreased by $5.07 \mathrm{mg} / \mathrm{dL}$. The measured physiological parameters were not statistically significant between the two groups. In the KMC transport, there was a decreasing trend in the incidence of hypothermia, hypoglycemia, tachypnea, and tachycardia.

Conclusion. There are no significant differences in the heart rate, respiratory rate, temperature, oxygen saturation, and blood glucose levels among preterm neonates on KMC transport compared with preterm neonates on transport incubators, which is the current standard of care. The study showed that KMC transport is equally effective as a

Presented and won $1^{\text {st }}$ place Research Contest in the PSNBM $1^{\text {st }}$ International Conference and $7^{\text {th }}$ Annual Meeting 2013.

Presented as Poster in the Pediatric Academic Societies' Annual Meeting 2013.

Presented (Oral) in the $9^{\text {th }}$ International Conference Kangaroo Mother Care 2012.

Presented and won $1^{\text {st }}$ place in the UP-PGH, Department of Pediatrics Fellows' Research Presentation 2012.

Corresponding author: Renelyn P. Ignacio, MD

Newborn Intensive Care Unit

Department of Pediatrics

Baguio General Hospital and Medical Center

Baguio General Hospital Driveway

Baguio, Benguet, Philippines

Email: ren_ignacio_md@yahoo.com transport incubator. Hence, in low-resource settings, KMC transport may be used as a safe and effective neonatal transport.

Key Words: Kangaroo Mother Care, Incubator, Transport, Preterm

\section{INTRODUCTION}

Through the years, advances in Obstetrics and Neonatal Medicine have resulted in an increased number of premature infants who survive. At Philippine General Hospital (PGH) alone, the prematurity rate has increased from 101 to 142 preterm deliveries per 1000 deliveries from 2005 to $2009 .^{1}$

Limited human resources and access to high-technology neonatal care restrict us from providing all preterm 
neonates good quality health care safe, effective, accessible, and affordable.

The Kangaroo Mother Care (KMC), also known as skin-to-skin care, was developed out of necessity in 1979 in the Neonatal Intensive Care Unit (NICU) of Instituto Materno Infantil in Bogota, Colombia, by Dr. Edgar Rey Sanabria and Dr. Hector Martinez Gomez. It was used as a substitute for incubators for preterm neonates to address the shortage of caregivers and the lack of resources. It was initially defined as early, prolonged, and continuous skin-toskin contact between mother and low birth weight (LBW) newborn infant until the $40^{\text {th }}$ week of postnatal gestational age. ${ }^{2}$ At present, it is defined as a form of parental caregiving where the newborn low birth weight infant is intermittently nursed skin to skin in a vertical position between the mother's breast or against the father's chest for a nonspecific period. ${ }^{3}$

$\mathrm{KMC}$ is a scientifically sound, effective, and efficient alternative to neonatal care units in many settings. It delivers high-quality care at a fraction of the cost of usual care. ${ }^{4}$

The World Health Organization (WHO) stipulated that the benefits of $\mathrm{KMC}$ offer more than an alternative to incubator care, stating that it provides an effective way to meet infants' needs for warmth, breastfeeding, protection from infection, stimulation, safety, and love across all healthcare settings. ${ }^{4}$

Numerous studies on KMC, both community, and hospital-based, have proven safe and effective. According to these studies, benefits of KMC for preterm LBW neonates include better weight gain, reduced nosocomial infection and severe illness, earlier hospital discharge, provision of warmth, higher oxygen saturation, higher Brazelton Neonatal Behavioral Assessment Scale scores on orientation and state regulation, increased duration of sleep bouts, encourages breastfeeding and enhances milk production..$^{5-13}$

Furthermore, studies have shown that heart rate, respiratory rate, respiration, oxygenation, oxygen consumption, blood glucose, and behavior observed in preterm LBW neonates on KMC tend to be similar or better than those separated from their mothers. ${ }^{13,14}$

With the cited advantages of KMC on cardiorespiratory and temperature stability, the question is, can KMC be used as a means of neonatal transport in the immediate newborn period and provide the same benefits?

Transport incubators introduced in 1900 by Dr. Joseph De Lee have been used up to the present as transportation for neonates. A transport incubator is a mobile transparent box designed to keep the neonate warm during transport. Such equipment is beneficial in our institution, where the NICU complex is three floors away from the delivery room and may take about 10-15 minutes of transport. However, not all hospitals in developing countries like ours can acquire such expensive equipment. Moreover, using the transport incubator carries the risk of a higher incidence of intraventricular hemorrhage (IVH) caused by mechanical effects such as shaking or vibration of the head and the instability of temperature and blood pressure. ${ }^{15}$ If transporting the neonate in kangaroo position could maintain the temperature and cardiorespiratory stability of the neonate and address such risks. Then the money can be saved and diverted to other life-saving measures.

The literature search yielded only one study done by Sontheimer on KMC as a means of transporting neonates. ${ }^{15}$ It is for this reason that this study is being conducted.

\section{REVIEW OF LITERATURE}

Studies have shown the many physiologic outcomes of $\mathrm{KMC}$ found to be beneficial, especially to preterm neonates.

A study done by Bergman et al., which compared $\mathrm{KMC}$ and conventional incubators, showed that KMC results in better physiological outcomes and stability than the same care provided in closed servo-controlled incubators. ${ }^{16}$

Studies on healthy preterm neonates showed that during $\mathrm{KMC}$, they not only remain clinically stable but also show a more efficient gas exchange. In a study done by Fohe et al., the respiratory rate decreased by 5 per minute, oxygen saturation improved by $0.4 \%$, accompanied by an increase in transcutaneous $\mathrm{pO}_{2}$ of $4.8 \mathrm{mmHg}$ and a decrease in transcutaneous $\mathrm{pCO}_{2}$ by $1.2 \mathrm{mmHg}$ during $\mathrm{KMC} .{ }^{10}$

Another study done by Ludington-Hoe et al. showed that cardiorespiratory changes remained within clinically acceptable ranges during KMC, and no apnea, bradycardia, and periodic breathing were noted. ${ }^{17}$

A study done by Gale et al. showed that in infants 30-33 weeks gestational age or weighing 1.2 to $3 \mathrm{~kg}$, the temperature, pulse oxygenation, and respiratory rate remained within normal parameters during KMC. ${ }^{18}$

A study comparing paternal skin-to-skin (kangaroo care) and those placed on the cot showed that neonates on paternal skin-to-skin cried less, were calmer, and reached a drowsy state earlier than the neonates in the cot group. ${ }^{19}$

Regarding the neonatal risks of $\mathrm{KMC}$, a study by Bohnhorst et al. concluded that KMC was associated with a significant increase in the combined frequency of bradycardia and hypoxemia and with less regular breathing, which was related to heat stress. ${ }^{9}$ Hence, it is recommended that body temperature, heart rate, and oxygenation be monitored during KMC.

In another study done by Gale et al., preterm neonates less than 30 weeks gestational age or weighing less than $1.2 \mathrm{~kg}$ showed signs of restlessness, tachycardia, and decreased oxygenation during prolonged KMC. ${ }^{18}$

Neonatal transport is not suitable for neonates, for it entails risks, especially to those born prematurely. It adds stress and breaks the continuum of care. Neonatal transport is a procedure that demands the highest professionalism and planning and should only be undertaken in close cooperation with the unit receiving the infant. ${ }^{20}$ The physiologic changes in the preterm neonate must be taken into consideration. 
The preterm neonate has thin skin, increased transdermal water loss, absent subcutaneous fat, and absent brown fat, which predisposes to hypothermia, eventually leading to depletion of energy stores, protein breakdown, and acidosis sclerema, increased oxygen consumption, sepsis, and death. ${ }^{20}$ Neonatal transport predisposes the neonate to significant cold exposure. Evidence from studies on neonatal transport between wards within hospitals suggests that even in such controlled settings, the risk of hypothermia during transport is high. ${ }^{21}$ Keeping the neonate warm reduces the metabolic rate and oxygen consumption. One of the many ways to provide warmth is through KMC because the mother's body is an excellent heat source. ${ }^{20}$

Premature neonates are also prone to having apnea due to immaturity of the respiratory center, which usually responds to tactile stimulation. The caregiver's rhythmic breathing during $\mathrm{KMC}$ stimulates the neonate, thus reducing the occurrence of apnea. ${ }^{22}$

Limited exposure to antigens before birth renders the preterm neonate immunodeficient. KMC contributes to the immuno-protection of the neonate by stimulating the mucosa-associated lymphoid tissue system and colonization of the neonate with maternal skin flora; as well as to the protection of the neonate from hypoglycemia. ${ }^{23}$

In an article presenting description and preliminary data for KMC transport done by Sontheimer et al., heart rate, respiratory rate, oxygen saturation, and rectal temperature for both non-oxygen and oxygen- requiring neonates with ages of 1 hour to 79 days remained stable during KMC transport by either the mother, father, doctor or nurse lasting 10 to 300 minutes traveling to a distance of 2 to 400 $\mathrm{km} .{ }^{15}$ The study concluded that KMC transport might be considered a safe, effective, and inexpensive method.

\section{RESEARCH QUESTION}

This study aims to answer the question, "Will the use of KMC transport among physiologically stable preterm neonates ( $\leq 36$ weeks gestational age and weighing $\leq 2200$ grams) transported from the delivery room to the neonatal intensive care unit result in improved cardiorespiratory and temperature stability compared to those in transport incubators?"

\section{OBJECTIVES}

The main objective of this investigation is to determine the efficacy and safety of KMC transport for physiologically stable preterm neonates $\leq 36$ weeks and weighing $\leq 2200$ grams. Specifically, this study aims:

1. To determine if there will be a significant change in heart rate (decrease by five beats/min, respiratory rate (decrease by five breaths/min), oxygen saturation (increase by $5 \%$ ), and temperature (increase by $0.3^{\circ} \mathrm{C}$ ) after $\mathrm{KMC}$ transport compared to transport incubator;
2. To determine if KMC transport would result in adverse events such as temperature instability, cardiorespiratory instability, oxygen desaturation, restlessness, and intraventricular hemorrhages (IVH);

\section{METHODS}

\section{Study Design}

Over seven months, this prospective, non-blinded, randomized controlled trial was conducted in a tertiary level NICU at the Philippine General Hospital.

\section{Study Population}

The study population included 92 participants, 46 for each of the control and treatment arms. Recruitment of participants from September 10, 2011, to April 18, 2012, was done by a third person not directly involved in the study at the delivery room of the said hospital.

Eligible participants included all physiologically stable neonates, with an APGAR score of $\geq 7$ at the first and fifth minute of life, not requiring any ventilatory support, intravenous fluids, or vasopressors, and were admitted at the neonatal intensive care unit with gestational age $\leq 36$ weeks by pediatric aging and birth weight of $\leq 2200$ grams. The age of gestation and weight was chosen because it is the cut-off age of gestation and cut-off weight for NICU admission of the institution in which the study was done.

Exclusion criteria included preterm neonates needing any form of ventilatory support, intravenous fluids, vasopressors, chest tubes, and umbilical lines, and the presence of significant, life-threatening congenital abnormalities and fetal chromosomal anomalies. Preterm neonates whose caregivers had any signs or symptoms of infection such as fever, conjunctivitis, cough, colds, oral sores, and infected skin lesions were likewise excluded.

Among the 92 participants recruited, two dropped out of the study due to the development of respiratory distress before transport.

\section{Randomization}

As soon as informed consent was obtained from the mothers after delivery or during skin-to-skin contact, the participants were randomized into two groups: the intervention group (KMC transport) or the control group (transport incubator). Randomization was achieved using a computer-generated random list of numbers. Participants in the intervention group were transported from the delivery room to the neonatal intensive care unit on $\mathrm{KMC}$ transport. In contrast, the participants in the control group were placed in the transport incubator.

\section{Intervention Group}

For the safety of the participants, all persons involved in the study had KMC training. 
For the participants in the intervention group, the presence of their caregivers was essential for their transport. Aside from the informed consent from the mother, and informed consent from the caregiver was also obtained. The caregiver performed a brief history and physical examination, including the correct axillary temperature measurement using a 5-second digital thermometer with a beeper (Model: ST8B30). The caregiver in the intervention group was advised in detail about the KMC transport and was taught how to recognize danger signs such as apnea, cyanosis, and decreased activity in their neonates. The caregiver's chest was then wiped dry with a clean cloth. Avoidance of skin disinfectants allowed the possibility of colonization of caregiver's flora instead of hospital flora, thereby protecting the neonate from infection. Such skin-to-skin practice is also presently being implemented in the Essential Intrapartum and Newborn Care (EINC) protocol of the WHO. The caregiver had to perform the prescribed handwashing using $70 \%$ alcohol and was required to wear a sterile gown over a KMC tube blouse. A new gown and tube blouse were provided for every participant by the investigator. The caregiver was comfortably seated on a wheelchair while waiting for the neonate to be positioned, as shown in Figure 1.

The neonate was positioned following the WHO: Kangaroo Mother Care, A Practical Guide Handbook, where the neonate donned with a cap and diaper with the back covered with a receiving blanket was transferred gently and placed upright against the caregiver's bare chest with the

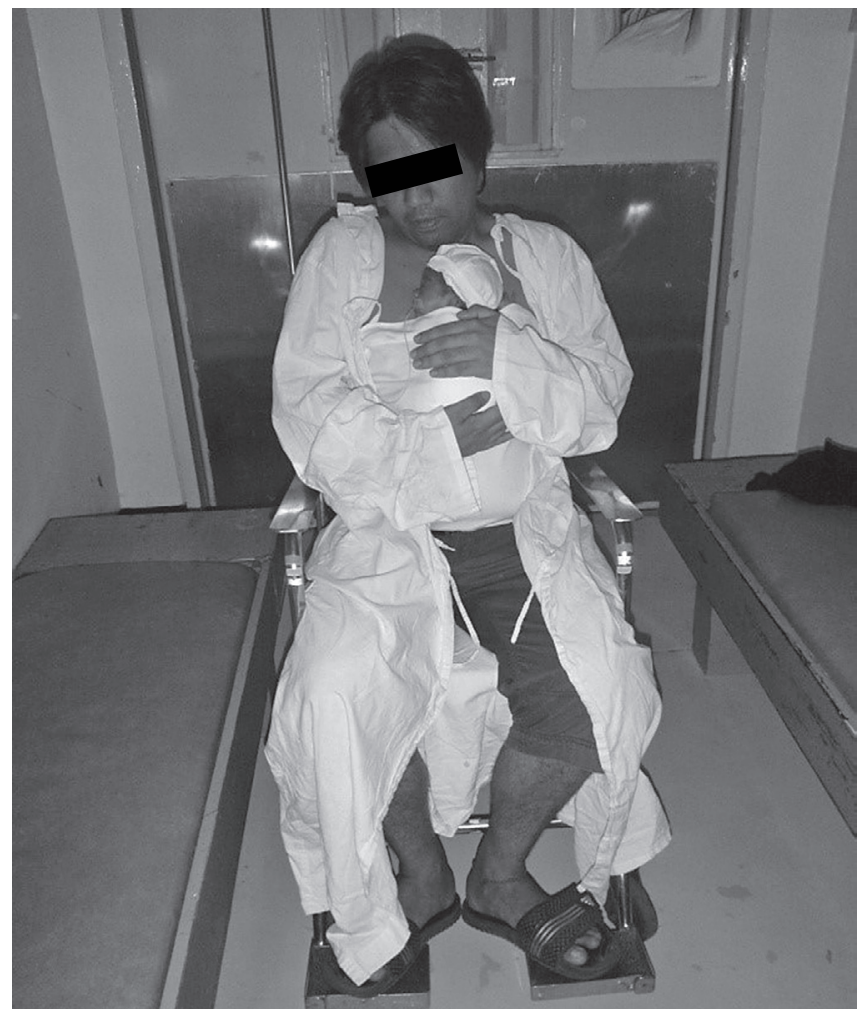

Figure 1.Kangaroo mother care transport. head turned to one side and in a slightly extended position to keep the airway open and to allow visualization of the neonate by the caregiver for observation of danger signs. The hip was flexed and abducted in a "frog-like" position. The arms were also bent. The chest and the abdomen were at the level of the caregiver's chest and epigastrium, respectively. The caregiver's rhythmic breathing stimulates the neonate, thus reducing the occurrence of apnea. ${ }^{22}$ After proper positioning of the neonate in kangaroo position, they were secured using the $\mathrm{KMC}$ tube blouse worn by the caregiver, who was instructed to support the back and the neck of the neonate with their hands during transport.

\section{Control Group}

The neonates in the control group were also donned with a cap and diaper. They were transported from the delivery room to the neonatal intensive care unit using a Medix TR-306 insulated double-walled transport incubator which ensured that the temperature of the inner wall of the incubator was not affected by a cooler room temperature, as shown in Figure 2. The transport incubator was already preheated at a neutral thermal environmental temperature of $34^{\circ} \mathrm{C}$. The preset temperature of $34^{\circ} \mathrm{C}$ was chosen to encompass the range of temperatures required by neonates weighing $\leq 2500$ grams and who were on the 0 to 6 hours of life (<1200 grams: $34-35.4^{\circ} \mathrm{C} ; 1200-1500$ grams: $33.9-$ $34.4^{\circ} \mathrm{C} ; 1501-2500$ grams $\left.32.8-33.8^{\circ} \mathrm{C}\right) .{ }^{24}$

\section{Monitoring}

Participants in both groups were monitored for cardiorespiratory instability, desaturations, hypothermia,

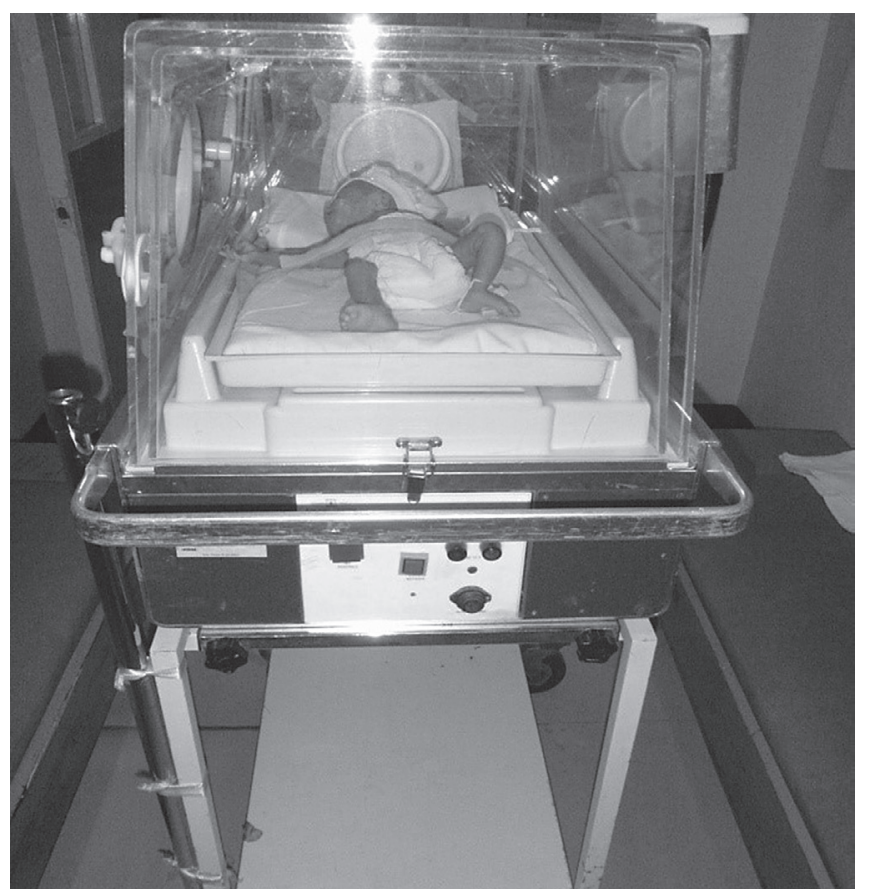

Figure 2.Transport incubator. 
and hypoglycemia. This was done through measurement of heart rate, respiratory rate, temperature, and oxygen saturation of the participants taken before transfer to KMC transport or transport incubator (baseline), 15 minutes while on KMC transport or transport incubator (pre-transport), immediately on placing the infant on the preset $34^{\circ} \mathrm{C}$ radiant warmer upon arrival at NICU (on arrival) and 15 minutes after arrival at NICU (post-transport). Capillary blood glucose was taken pre-transport and post-transport.

The heart rate, respiratory rate, and oxygen saturations were measured when the participant was at rest or was calm and quiet to avoid significant changes.

To avoid stimulating the neonate, the first vital sign being measured was the respiratory rate. The chest rise was counted for one minute using a pocket watch with a second hand.

A portable finger probe pulse oximeter with a $\mathrm{SpO}_{2}$ measuring range of $0-100 \%$ and a pulse rate measuring range of 30 to $250 \mathrm{bpm}$ was used to measure the participant's heart rate and oxygen saturation. The pulse oximeter was cleaned with $70 \%$ alcohol before placing it on the participant's right hand. The reading was done after a minute of putting it or when the participant becomes calm or quiet, whichever comes first.

The pulse oximeter is maintained from the initial heart rate and oxygen saturation measurement until the $15^{\text {th }}$ minute after arrival at NICU.

The temperature is measured using a 5 -second digital thermometer with a beeper (Model: ST8B30) placed on the left axillary area of the participant. The thermometer was cleaned with $70 \%$ alcohol before use. The tip of the thermometer was placed securely in the left armpit of the participant and was held down tightly at their side until the digital thermometer beeped. The thermometer was removed when the Celsius sign in the LCD stopped blinking.

If the baseline temperature was less than $36.5^{\circ} \mathrm{C}$, specific measures such as putting the neonate on skin-to-skin contact with the mother or placing the neonate under the radiant warmer or droplight were done to restore the normal temperature. Once the temperature was normal, a repeat measurement of the respiratory rate, heart rate, and $\mathrm{SPO}_{2}$ was taken to ensure that these were within normal ranges before transfer to kangaroo transport or transport incubator.

Lastly, the capillary blood glucose (CBG) was measured using the One Touch Ultra blood glucose meter and strip. Participants were pricked in the right heel for the collection of the blood sample.

If the blood glucose was $<50 \mathrm{mg} / \mathrm{dL}$ before transport, the participant was either given back to the mother for breastfeeding or $2 \mathrm{cc} / \mathrm{kg}$ of $\mathrm{D} 10 \mathrm{~W}$ via the intravenous route. Transport proceeded when the repeat CBG showed normal results.

As soon as all vital signs were within normal range, participants in both groups were transported from the delivery room to the NICU, three floors away. The travel took about 5-20 minutes. They were accompanied by a pediatric resident equipped with resuscitation materials during transport. There were no adverse events such as accidents from transferring infant to kangaroo position; neither was apnea, cyanosis, bradycardia, tachycardia, and decreased oxygen saturation during transport. Monitoring for adverse events was continued up to the first hour after transport.

Recording of data using a standard database was done. Routine medical management for preterm neonates and their medical complications was provided during the study as deemed by the attending physician.

\section{Definition of Terms}

- Extremely low birth weight neonates are neonates weighing $<1000$ grams.

- KMC transport is a means of transportation. The low birth weight physiologically stable newborn is placed skin-to-skin in a vertical position against the father's or any caregiver's chest during transport from the delivery room to the neonatal intensive care unit.

- Transport incubator is a mobile transparent box designed to keep the neonate warm during transport.

- Physiologically stable neonates are neonates with stable vital signs (heart rate of $100-160$ beats per minute, respiratory rate of 30-60 breaths per minute, the temperature of $36.5^{\circ} \mathrm{C}-37.5^{\circ} \mathrm{C}$, and oxygen saturation of $88 \%$ and above at the $15^{\text {th }}$ minute of life)

- Preterm neonates are neonates born before 37 weeks of gestation.

- Very low birth weight neonates are neonates weighing 1000-1500 grams.

- Caregiver: the father or any relative willing to do KMC transport.

\section{Statistical Analysis}

A sample size of 46 subjects for each the control and intervention arm was calculated to achieve a power of $95 \%$ and an alpha error of 0.05 based on the study of Suman Rao et al. on the secondary outcome of the effect of KMC on morbidities (hypothermia). ${ }^{25}$

Data were recorded on a predesigned template, tabulated, and statistically analyzed.

For the baseline characteristics of participants, categorical data were analyzed statistically by the Chi-square test. In contrast, the T-test and Fisher's exact test were used to compare quantitative measurements.

The mean change on participants' physiologic responses in heart rate and temperature pre-transport and upon arrival at the NICU were analyzed using the independent T-test. In contrast, the mean change on the physiologic responses in respiratory rate, oxygen saturation, and capillary blood glucose was analyzed using the Wilcoxon-MannWhitney test. A p-value of $<0.05$ was considered significant.

The results obtained for adverse events (hypothermia, hyperthermia, tachycardia, apnea, bradypnea, tachypnea, oxygen desaturation, hypoglycemia, hyperglycemia, and 
intraventricular hemorrhage), as well as heart rate, respiratory rate, temperature, and oxygen saturation measurements pre-transport, upon arrival at NICU and post- transport, were discussed based on exploratory analysis (descriptive measurements, graphs).

\section{Limitation of the Study}

The limitation of the study is that it is a non-blinded randomized controlled trial.

\section{Ethical Consideration}

The study with PGH ERB Registration number of PED 2011-05-06-064 received ethical approval from the Philippine General Hospital Expanded Hospital Research Office (EHRO) before its conduction on July 11, 2011. Written informed consent from the conscious and coherent mother was taken after delivery or during skin-to-skin contact with her baby and from the caregiver if the neonate belonged to the KMC transport group after a thorough explanation of the study. A copy of the consent was given to them. Participation was voluntary, and withdrawal anytime during the investigation was respected. Confidentiality of the participant's database was also ensured.

The study protocol was submitted to KMC Foundation, Philippines, Inc. to fund the study and was approved on September 13, 2011.

The KMC Foundation, Philippines, Inc. is a nonprofit organization established in 2008. It is composed of a recognized group of professionals committed to embracing every newborn's right to a healthy life through Kangaroo Mother Care by 1) using creative training, education, and development, 2) advocating its adoption as the standard of care for low-birth-weight infants and 3) nurturing the infant by involving the family and the community. The Foundation's goal is to develop, monitor, and accredit KMC centers and encourage the continued application of $\mathrm{KMC}$ as a standard practice in neonatal care. To meet such goals, one of its advocacies is to promote and support local researches that can improve knowledge and practices of $\mathrm{KMC}$, a highimpact low- technology strategy that can help decrease neonatal mortality.

\section{RESULTS}

A total of 92 participants were recruited; however, two dropped out due to the development of respiratory distress before transport. The final study groups comprised 45 neonates in the KMC transport and 45 neonates in the transport incubator.

Table 1 shows there was no significant difference in the maternal risk factors in both groups. Among the 45 participants in the $\mathrm{KMC}$ transport group, $55.6 \%$ were females, while the transport incubator group had $46.7 \%$ females. For both groups, $53.3 \%$ of the participants were born via spontaneous vaginal delivery, while $46.7 \%$ were born via cesarean section. Participants in the KMC transport had a mean gestational age of 34.9 weeks, mean weight of 1765.6 grams, and APGAR score of 8.8 and 9 at 1 and 5 minutes of life as compared to a mean gestational age of 35 weeks, mean weight of 1820 grams and APGAR score of 8.8 and 8.9 at 1 and 5 minutes of life for those in the transport incubator.

Baseline characteristics of the participants are described in Table 2. Participants in the KMC transport had a mean age of 167.3 minutes upon enrolment to the study compared to 169.2 minutes in the transport incubator group. The mean baseline temperature for both groups was the same at $36.3^{\circ} \mathrm{C}$. Caregivers aged 17 to 58 years old with baseline temperatures ranging from 36 to $37.1^{\circ} \mathrm{C}$ served as transport incubators for the participants in the KMC transport. The mean duration of transport from the delivery room to the neonatal intensive care unit was 8.3 minutes. Transport incubators used by the participants in the control group had a baseline neutral thermal environmental temperature ranging from 33.1 to $34.5^{\circ} \mathrm{C}$. The mean duration of transport from the delivery room to the neonatal intensive care unit was 7.4 minutes. Statistical analysis on the baseline characteristics of participants showed no significant differences in any of the variables measured.

As seen in Figure 3, the mean heart rate of participants in both groups from baseline to pre-transport decreased by 2.1 beats per minute in the $\mathrm{KMC}$ transport group (intervention) and two beats per minute in the transport incubator group (control). The mean heart rate increased by 1.6 beats per minute upon arrival at the NICU, then decreased by 4.6 beats per minute 15 minutes post-KMC transport. In contrast, the transport incubator group registered a decrease in the heart rate by 2.4 beats per minute upon arrival and then by 2.5 beats per minute 15 minutes post- transport incubator.

As for the respiratory rate, Figure 4 shows that in the KMC transport group, pre-transport value decreased by 0.29 breaths/minute from baseline, increased by 0.18 breaths/ minute upon arrival at the NICU, and decreased by 0.98 breaths/minute post-transport. For the transport incubator group, the respiratory rate increased by 1.98 breaths/ minute from baseline to pre-transport and 0.64 breaths/ minute from pre-transport to arrival at NICU. However, it decreased to 1.13 breaths/minute post-transport.

Figure 5 shows the same baseline mean temperature of $36.32^{\circ} \mathrm{C}$ for neonates in both groups. Pre-transport, there was a noted increase by $0.34^{\circ} \mathrm{C}$ in the $\mathrm{KMC}$ transport group and $0.38^{\circ} \mathrm{C}$ in the transport incubator group. Upon arrival, there was a reported decrease by $0.01^{\circ} \mathrm{C}$ in the $\mathrm{KMC}$ transport group and $0.1^{\circ} \mathrm{C}$ in the transport incubator group. Post-transport, an increase was noted by $0.04^{\circ} \mathrm{C}$ in the $\mathrm{KMC}$ transport group and a decrease by $0.02^{\circ} \mathrm{C}$ in the transport incubator group.

As shown in Figure 6, the mean oxygen saturation in the KMC transport group showed an increasing trend from baseline to post-transport. It increased by $0.58 \%$ from baseline to pre-transport, $0.07 \%$ from pre-transport to 
Table 1. Baseline characteristics of participants

\begin{tabular}{|c|c|c|c|}
\hline Characteristics & KMC transport $(n=45)$ & Control $(n=45)$ & p-value \\
\hline \multicolumn{4}{|l|}{ Maternal Risk Factors (\%) } \\
\hline Maternal Age (mean \pm SD) & $27.8 \pm 7.5$ & $28.0 \pm 7.1$ & $0.94^{t}$ \\
\hline Gestational HPN & 4.4 & 2.2 & $1.00^{f}$ \\
\hline Chronic HPN & 11.1 & 6.7 & $0.71^{\dagger}$ \\
\hline Pre-eclampsia & 15.6 & 8.9 & $0.33^{c}$ \\
\hline Eclampsia & 2.2 & 6.7 & $0.62^{f}$ \\
\hline Hepa B & 0 & 4.4 & $0.49^{f}$ \\
\hline Multiple gestations & 17.8 & 35.6 & $0.06^{c}$ \\
\hline HELLP syndrome & 2.2 & 2.2 & $1.00^{f}$ \\
\hline Gestational DM & 6.7 & 2.2 & $0.62^{f}$ \\
\hline PROM>12 & 11.1 & 6.7 & $0.71^{f}$ \\
\hline Acquired pneumonia & 0 & 6.7 & $0.24^{f}$ \\
\hline Placenta previa & 2.2 & 4.4 & $1.00^{f}$ \\
\hline UTI & 2.2 & 4.4 & $1.00^{f}$ \\
\hline Oligohydramnios & 4.4 & 2.2 & $1.00^{f}$ \\
\hline Young Primigravida & 6.7 & 4.4 & $1.00^{f}$ \\
\hline Steroids use (\%) & 57.8 & 64.4 & $0.52^{c}$ \\
\hline Age of Gestation (weeks) (mean \pm SD) & $34.9 \pm 1.6$ & $35.0 \pm 1.2$ & $0.82^{\mathrm{t}}$ \\
\hline Birthweight (grams) (mean \pm SD) & $1765.6 \pm 327.1$ & $1820.0 \pm 253.3$ & $0.38^{\mathrm{t}}$ \\
\hline \multicolumn{4}{|l|}{ Sex; n (\%) } \\
\hline Male & $20(44.4)$ & $24(53.3)$ & $0.40^{c}$ \\
\hline Female & $25(55.6)$ & $21(46.7)$ & \\
\hline Manner of delivery; $\mathrm{n}(\%)$ & & & $1.00^{c}$ \\
\hline SVD & $24(53.3)$ & $24(53.3)$ & \\
\hline LSCS & $21(46.7)$ & $21(46.7)$ & \\
\hline Apgar score (1 $\left.{ }^{\text {st }} \min \right)($ mean $\pm S D)$ & $8.8 \pm 0.6$ & $8.8 \pm 0.5$ & $0.56^{t}$ \\
\hline Apgar score (5 $\left.5^{\text {th }} \min \right)($ mean $\pm S D)$ & $9.0 \pm 0.1$ & $8.9 \pm 0.3$ & $0.41^{\mathrm{t}}$ \\
\hline
\end{tabular}

Table 2. Baseline characteristics of participants on transport

\begin{tabular}{lccc}
\multicolumn{1}{c}{ Characteristics } & KMC transport $(\mathbf{n}=\mathbf{4 5})$ & Control $(\mathbf{n}=\mathbf{4 5})$ & p-value \\
\hline Age at transport (minutes) (mean \pm SD) & $167.3 \pm 71.6$ & $169.1 \pm 75.2$ & $0.91^{\mathrm{t}}$ \\
\hline Initial temperature $\left({ }^{\circ} \mathrm{C}\right)($ mean $\pm \mathrm{SD})$ & $36.3 \pm 0.5$ & $36.3 \pm 0.6$ & $1.00^{\mathrm{t}}$ \\
\hline Duration of Transport (minutes) (mean \pm SD) & $8.3 \pm 2.8$ & $7.4 \pm 1.9$ & $0.09^{\mathrm{t}}$ \\
\hline
\end{tabular}

arrival at NICU, and $0.26 \%$ from NICU to post-transport. Compared to the transport incubator group, there was a noted increase by $0.09 \%$ from baseline to pre-transport and $0.84 \%$ from pre-transport to arrival at NICU, then a decrease by $0.13 \%$ from arrival at NICU to post-transport.

There were no significant changes in the heart rate, respiratory rate, temperature, and oxygen saturation between the two groups.

The mean changes in the physiologic responses (heart rate, respiratory rate, temperature, oxygen saturation, and capillary blood glucose) of participants according to the group between pre-transport and upon arrival are compared in Table 3. The mean change in heart rate in the KMC transport group decreased by 1.6 beats per minute instead of increasing heart rate by 2.4 beats per minute in the transport incubator group. The mean change in the respiratory rates for both groups decreased by 0.18 breaths per minute in the $\mathrm{KMC}$ transport group and 0.64 breaths per minute in the control group. For the mean change in temperature, there was a noted increase in the $\mathrm{KMC}$ transport group by $0.01^{\circ} \mathrm{C}$ compared to a decrease by $0.02^{\circ} \mathrm{C}$ in the transport incubator group. The mean change in oxygen saturation for both groups showed a decline of $0.07 \%$ and $0.84 \%$ for the $\mathrm{KMC}$ transport group and transport incubator group, respectively. Also, the mean change in the blood glucose level decreased by $5.07 \mathrm{mg} / \mathrm{dL}$ in the $\mathrm{KMC}$ transport group compared to $4.6 \mathrm{mg} / \mathrm{dL}$ in the transport incubator group. Statistical analysis on the mean change on the physiologic responses (heart rate, respiratory rate, temperature, oxygen saturation, and capillary blood glucose) of participants pre-transport 
Table 3. Mean change on the physiologic responses of participants(pre-transport and upon arrival) according to group

\begin{tabular}{lccc}
\hline \multicolumn{1}{c}{ Characteristics } & KMC transport $(\mathbf{n}=\mathbf{4 5})$ & Control $(\mathbf{n}=\mathbf{4 5})$ & p-value \\
\hline Heart rate $($ mean \pm SD) & $-1.6 \pm 9.6$ & $2.4 \pm 11.5$ & 0.07 \\
\hline Respiration & $-0.18 \pm 5.7$ & $-0.64 \pm 4.3$ & 0.44 \\
(mean \pm SD) & 0.0 & -1.0 & \\
Median & -26 & -10 & \\
Minimum & 11 & 8 & \\
Maximum & & & \\
\hline Temperature (mean \pm SD) & $0.01 \pm 0.22$ & $-0.02 \pm 0.21$ & 0.53 \\
\hline O $_{2}$ sat & & & 0.19 \\
(mean \pm SD) & $-0.07 \pm 2.6$ & $-0.84 \pm 2.6$ & \\
Median & 0.0 & 0.0 & \\
Minimum & -5 & -8 & \\
Maximum & 9 & 5 & \\
\hline CBG & & & \\
(mean \pm SD) & $-5.07 \pm 21.4$ & $-4.6 \pm 21.5$ & \\
Median & -7.0 & -6.0 & \\
Minimum & -51 & -80 & \\
Maximum & 64 & 45 & \\
\hline
\end{tabular}

Wilcoxon-Mann-Whitney test (RR, $\mathrm{O}_{2}$ sat, $\mathrm{CBG}$ )

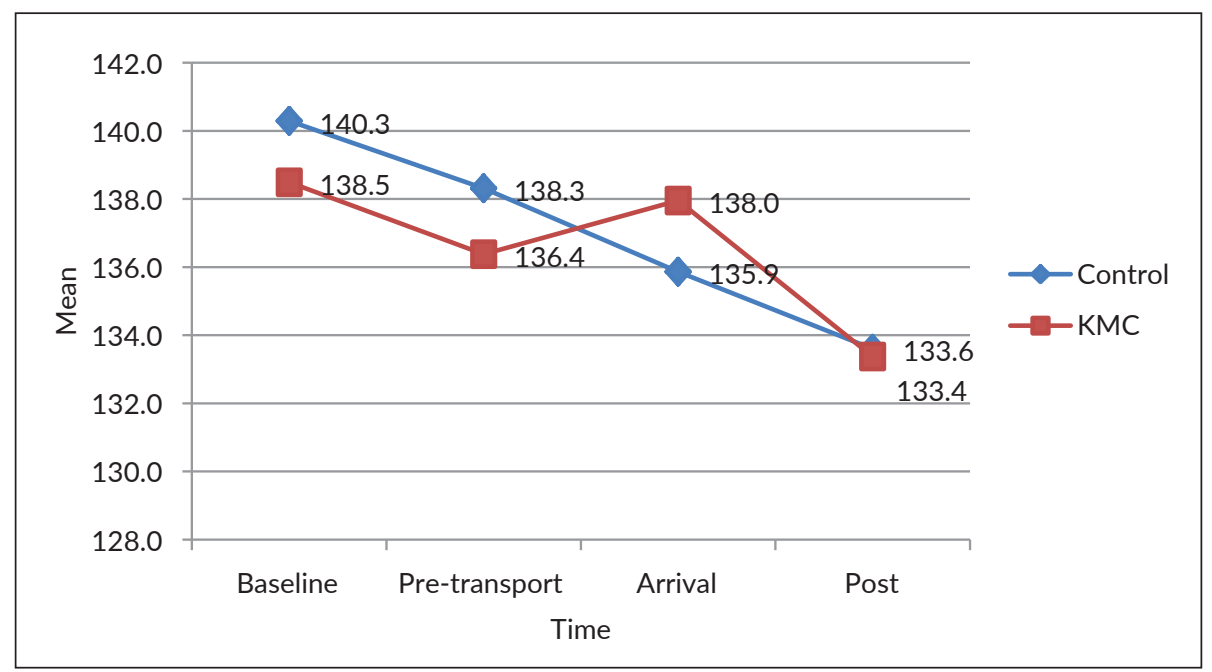

Figure 3.Mean heart rate of participants by time and group.

and upon arrival showed no significant differences between the two groups being compared.

In addition, there was a trend to a higher incidence of hypothermia at $24.44 \%$ (11 participants), hypoglycemia at $11.11 \%$ (5 participants), tachypnea at $8.89 \%$ (4 participants), and tachycardia at $6.67 \%$ (3 participants) in the transport incubator group compared to $11.11 \%$ (5 participants) of hypothermia, $6.67 \%$ (3 participants) of hypoglycemia and $2.2 \%$ (1 participant) of tachypnea and tachycardia in the KMC transport group but is not statistically significant. One participant in each group had hyperglycemia. In the KMC transport group, 1 participant had bradypnea with oxygen desaturation, while none was observed in the transport incubator group.

\section{DISCUSSION}

The transition from intrauterine to extrauterine life requires fundamental changes in the newborn's circulatory, respiratory, metabolic, and immune functions. ${ }^{20}$ And when these changes are coupled with neonatal transport, it would render the newborn, especially preterm neonates, to significant stress and be vulnerable to cardiorespiratory and temperature instability. 


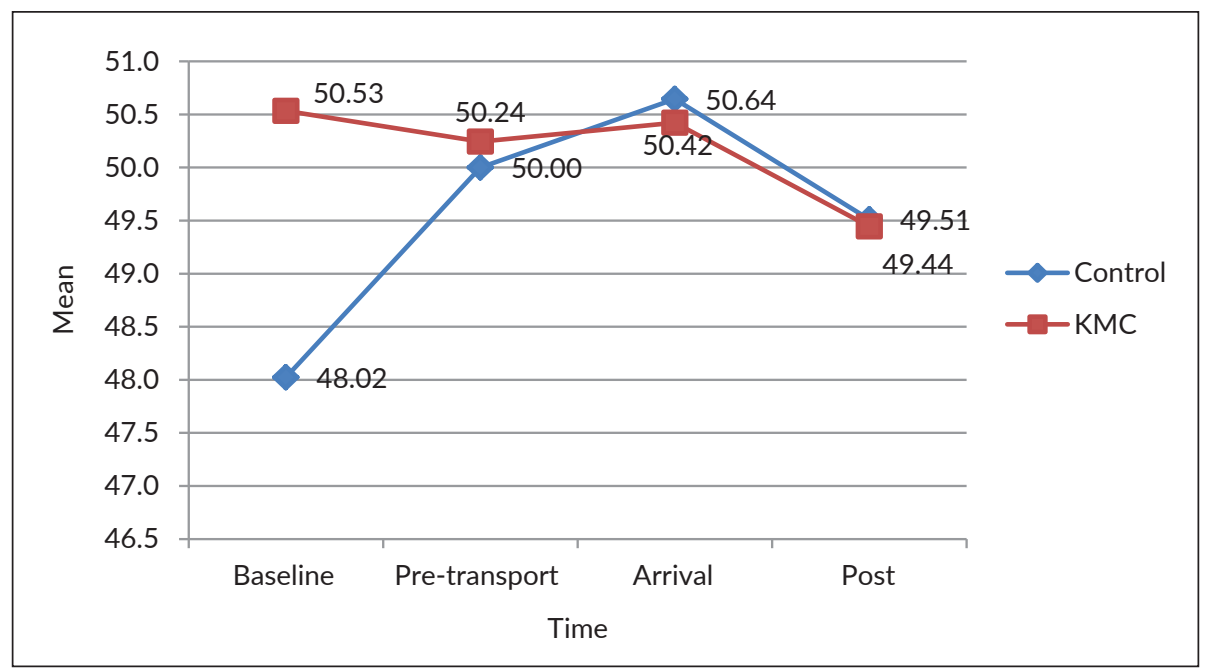

Figure 4.Mean RR of participants by time and group.

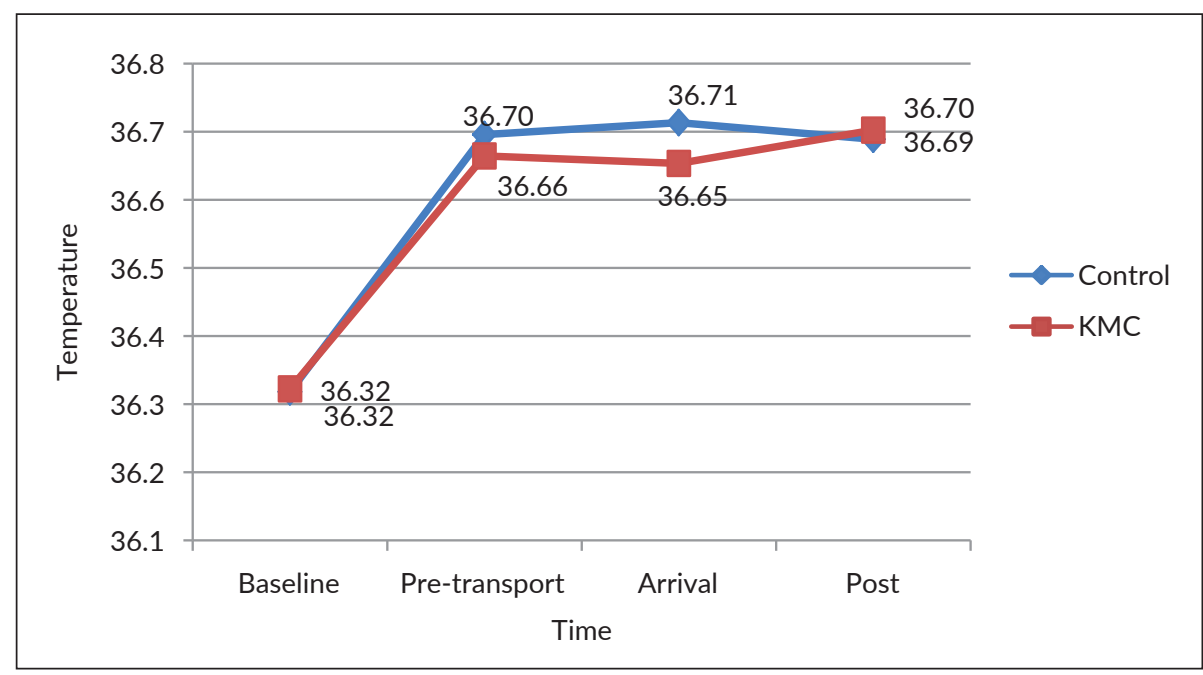

Figure 5.Mean temperature of participants by time and group.

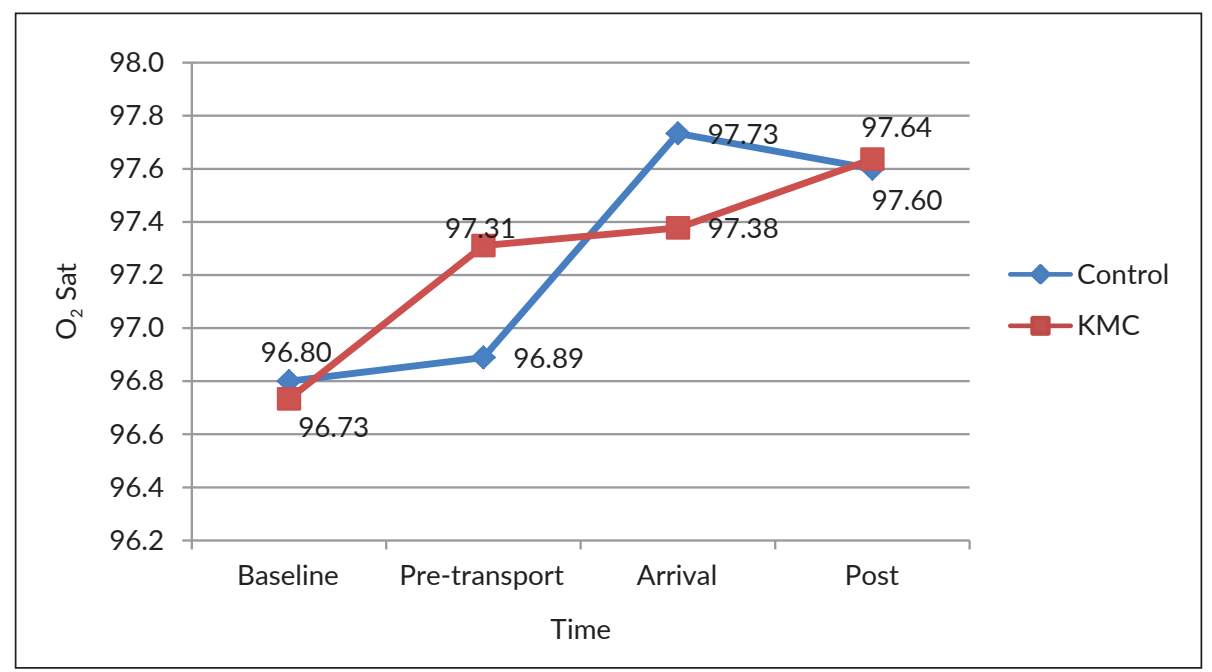

Figure 6.Mean $\mathrm{O}_{2}$ Sat of participants by time and group. 
Although KMC has been implemented in several developing and developed countries with promising outcomes on preterm neonates' cardiorespiratory and temperature stability, only one study has documented KMC transport as an alternative for transport incubators. ${ }^{15}$

The preliminary results of the descriptive study by Sontheimer et al. showed that KMC transport of stable preterm neonates could be performed over a short or long distance without compromising the infants' physiologic stability. ${ }^{15}$ The present study confirms this finding. The participants in the $\mathrm{KMC}$ transport group remained clinically stable with insignificant changes in heart rate, respiratory rate, oxygen saturation, and temperature post-transport.

Although not significant in this study, the decrease in the mean heart rate after KMC transport may have occurred because the neonates were calm and comfortable. The upright position of the neonate can explain the reduction of the respiratory rate seen. Ventilation is gravity-dependent, so an upright position optimizes respiratory function. ${ }^{26}$ A significant decrease in the respiratory rate in infants receiving Kangaroo Mother Care was evident in Acholet et al., Bauer et al., and Fohe et al.,10,27 However, this was not significantly different in this study.

Previous studies ${ }^{10,27,28}$ have shown that oxygen saturation levels have been found to increase by 2 to $3 \%$ during $\mathrm{KMC}$ compared to incubator values, and statistically significant increases have been confirmed by meta-analysis. ${ }^{29}$ An improvement in tissue oxygenation, as shown by the increased saturation, may have occurred because the newborns were calm and comfortable, which probably decreased oxygen consumption. ${ }^{30}$ In this study, both groups had a decrease in oxygen saturation, with the control group having a greater drop, though not significant compared to the $\mathrm{KMC}$ transport group.

A meta-analysis of 23 studies of 190 term and 326 preterm infants (gestational age range 26 to 36 weeks) concluded an increase in body temperature of $0.22^{\circ} \mathrm{C}$ during periods of KMC. ${ }^{31}$ In addition, studies done by Bauer et al. and Ludington-Hoe et al. have shown a significant reduction in episodes of hypothermia due to the provision of naturally modulated warmth while on skin-to-skin with the mother. ${ }^{14,32}$ However, this study showed no significant difference in the temperature between the two groups.

Preterm neonates are at high risk of having hypoglycemia because of inadequate glycogen sources. Only one study ${ }^{25}$ has documented that KMC reduces the frequency of hypoglycemia over the first 90 minutes after birth, although no explanation regarding this finding has been cited. In the present study, there was a higher incidence of hypoglycemia in the control group than in the intervention group.

\section{CONCLUSION AND RECOMMENDATION}

From the results obtained from this study, it can be suggested that preterm physiologically stable neonates on
KMC transport maintained their cardiorespiratory and temperature stability within clinically acceptable ranges with the occurrence of some adverse events, which is no different from those on transport incubator.

There are no significant differences in the heart rate, respiratory rate, temperature, oxygen saturation, and blood glucose levels among preterm neonates on KMC transport compared with preterm neonates on transport incubators, which is the current standard of care. The study showed that $\mathrm{KMC}$ transport is equally effective as a transport incubator. Hence, in low-resource settings, KMC transport may be used as a safe and effective neonatal transport.

To provide a more robust conclusion, a similar study with a larger population is recommended.

\section{Statement of Authorship}

Both authors participated in the collection of data and analysis and approved the final version submitted.

\section{Author Disclosure}

Both authors declared no conflicts of interest.

\section{Funding Source}

The study was funded by the Kangaroo Mother Care Foundation Philippines, Inc.

\section{REFERENCES}

1. Department of Pediatrics, University of the Philippines, Philippine General Hospital, Section of Newborn Medicine. Annual Perinatal Statistics. Unpublished data. 2005-2009.

2. Black K. Kangaroo Care and the Ventilated Neonate. Infant Journal. 2005; 1(4):127-32.

3. Pelaez J, Charpak N, Cuervo N. Kangaroo Mother Care, an Example to Follow from Developing Countries. BMJ. 2004 Nov; 329 (7475): 1179-81.

4. World Health Organization (WHO). Thermal Control of the Newborn: A Practical Guide. Maternal Health and Safe Motherhood Program 1997; WHO Geneva

5. Conde-Agudelo A, Diaz-Rossello JL, Belizan JM. Kangaroo Mother Care to Reduce Morbidity and Mortality in Low Birth Weight Infants (update of Cochrane Database Syst Rev 2000;(4): CD002771; PMID:11034759). Cochrane Database Syst Rev 2003; 3(2):CD002771, 1-23.

6. Paul VK, Ramanathan K, Deorari AK. Physiological and Clinical Benefits of Kangaroo Mother Care: Impressions from a Tertiary Care Newborn Nursery in Delhi. Indian J Pediatr. 2001 Jan; 68(11):85-9.

7. Bauer K, Uhrig C, Sperling P, Pasel K, Wieland C, Versmold HT. Body Temperatures and Oxygen Consumption during Skin-toskin (kangaroo) Care in Stable Preterm Infants Weighing Less than 1500 Grams. J Pediatr. 1997 Feb; 130(2): 240-4.

8. Ludington-Hoe SM, Nguyen N, Swinth JY, Satyshur RD. Kangaroo Care Compared to Incubators in Maintaining Body Warmth in Preterm Infants. Biol Res Nurs. 2000; 2(1):60-73.

9. Bohnhorst B, Heyne T, Peter CS, Poets CF. Skin-to-skin (kangaroo) Care, Respiratory Control, and Thermoregulation. J Pediatr. 2001 Feb; 138(2):193-7.

10. Fohe K, Skropf S, Avenarius S. Skin-to-skin Contact Improves Gas Exchange in Premature Infants. J Perinatol. 2000 Jul- Aug; 20(5): 311-5.

11. Ohgi S, Fukuda M, Moriuchi H, Kusumoto T, Akiyama T, Nugent JK, et al. Comparison of Kangaroo Care and Standard Care: Behavioral 
Organization, Development, and Temperament in Healthy, Low Birth Weight Infants through 1 Year. J Perinatol. 2002 Jul-Aug; 22(5):374-9.

12. Ludington-Hoe SM, Swinth JY. Developmental Aspects of Kangaroo Care. J Obstet Gynecol Neonatal Nurs. 1996 Oct; 25(8): 691-703.

13. Whitelaw A, Heisterkamp G, Sleath K, Acolet D, Richards M. Skin-to-skin Contact for Very Low Birth Weight Infants and Their Mothers. Arch Dis Child. 1998 Nov; 63 (11):1377-81.

14. Ludington-Hoe SM, Hadeed AJ, Anderson GC. Physiological Response to Skin-to-skin Contact in Hospitalized Premature Infants. J Perinatol. 1991 Mar; 11(1):19-24.

15. Sontheimer D, Fischer C, Buch K. Kangaroo Transport Instead of Incubator Transport. Pediatrics. 2004 April; 113(4): 920-3.

16. Bergman NJ, Linley NN, Fawcus SR. Randomized Controlled Trial of Skin-to-skin Contact from Birth versus Conventional Incubator for Physiological Stabilization in 1200-to 2199-gram Newborns. Acta Paediatr. 2004 June; 93(6):779-85.

17. Ludington-HoeSM, Anderson GC, Swinth JY, Thompson C, Hadeed AJ. Randomized Controlled Trial of Kangaroo Care: Cardiorespiratory and Thermal Effects on Healthy Preterm Infants. Neonatal Netw. 2004 May-Jun; 23(3):39-48.

18. Drosten- Brooks F. Kangaroo Care: Skin-to-skin Contact in the NIVU. MCN Am J Matern Child Nurs. 1993 Sep-Oct;18(5):250-3.

19. Erlandsson K, Dsilna A, Fagerberg I, Christensson K. Skin-to-skin Care with the Father after Cesarean Birth and Its Effect on Newborn Crying and Prefeeding Behavior. Birth. 2007 Jun; 34(2):105-14.

20. Hadley L, Lakhoo K. Neonatal Physiology and Transport. Pediatric Surgery. Second edition. Springer. 2020; Chapter 2: 15-24.

21. Daga AS, Daga SR, Patole SK. Determinants of Death among Admissions to Intensive Care Unit for Newborns. J Trop Pediatr. 1991; 37(2):53-6.

22. Thukral A, Chawla D, Agarwal R, Deorari AK, Paul VK. Kangaroo Mother Care an Alternative to Conventional Care. Indian J Pediatr. 2008 May; 75(5): 497-503.
23. Mantaring J. Lecture on Essential Newborn Care: The Evidence. Launching of the Essential Newborn Care. Sofitel Philippine Plaza. 2009 Dec.

24. Klaus MH, Fanaroff AF. Care of the High-Risk Neonate. WB Saunders. 5th edition; 2001:138.

25. Rao S, Udani R, Nanavati R. Kangaroo Mother Care for Low Birth Weight Infants: a Randomized Controlled Trial. Indian Pediatr. 2008 Jan; 45(1):17-23.

26. Ludington-Hoe S, Morgan K, Abouelfettoh A. A Clinical Guideline for Implementation of Kangaroo Care with Premature Infants of 30 or more Weeks' Postmenstrual Age. Advances in Neonatal Care. 2008 June; 8(3): S3-S23.

27. Acolet D, Sleath K, Whitelaw A. Oxygenation, Heart Rate, and Temperature in Very Low Birth Weight Infants during Skinto-skin Contact with Their Mothers. Acta Paediatr Scand. 1989 Mar; 78(2):189-93.

28. Ferreira C, Ludington S. Does Frequent Skin Contact Improve Respiratory Pattern in Preemies?. Respir Care. 1994; 39(11):1061.

29. Ludington-Hoe SM, Dorsey SG. Meta-analysis of Kangaroo Care Effects. J Invest Med. 1998; 46(1):175A.

30. Mohammadzadeh A, Farhat A, Jafarzadeh, M, Hasanzadeh L, Esmaeli, H. Advantages of Kangaroo Mother Care in Less than 2000 Grams Low Birth Weight Neonates. Medical Journal of the Islamic Republic of Iran. 2011; 25(1): 11-5.

31. Mori R, Khanna R, Pledge D, Nakayama T. Meta-analysis of Physiological Effects of Skin-to-skin Contact for Newborns and Mothers. Pediatr Int. 2010 Apr; 52(2):161-70.

32. Bauer K, Uhrig C, Sperling P, Pasel K, Wieland C, Versmold HT. Body Temperatures and Oxygen Consumption during Skin-to-skin (Kangaroo) Care in Stable Preterm Infants Weighing Less than 1500 Grams. J Pediatr 1997;130:240-4.

\section{The Acta Medica Philippina is now accepting limited advertising for its front and back cover (colored), as well as for available spaces in some of its pages, as appropriate. For inquiries and submission of proposals, please email us at actamedicaphilippina.upm@up.edu.ph}

